\title{
Investigating the quality and completeness of medication data available within the Indiana Network for Patient Care
}

\author{
Jaibir Khera ${ }^{1}$, Shaun Grannis ${ }^{1,2}$, Suranga Kasthurirathne $e^{1,2}$ \\ ${ }^{1}$ Indiana University School of Medicine, ${ }^{2}$ Center for Biomedical Informatics, Regenstrief Institute
}

\begin{abstract}
Background and Hypothesis: Healthcare data is increasingly fragmented across multiple points of care due to increased specialization of healthcare services and mobility of patient populations [1]. The Indiana Network for Patient Care (INPC) plays a pivotal role in capturing, standardizing, and integrating comprehensive datasets[2]. Currently, the INPC receives medication data from a variety of sources. However, some of these sources are costly and may be incomplete and/or inaccurate. We aim to characterize the degree to which additional claims data can augment or replace existing INPC medication data. Our hypothesis is that the new claims datasets will provide additional medication information for patients in the INPC.
\end{abstract}

Methods: Data analysts extracted and formatted a claims data sample for analysis. Individuals from the sample dataset were then mapped to INPC data using global person identifiers. The resulting analysis was split into two phases.

Phase 1: To provide an initial high-level assessment of overlap and complementarity between INPC and new claims data, we computed the number of patients captured in each data source. Patients were classified into five groups as illustrated in Figure 1. To investigate Medicare usage, we also stratified datasets by patient age: less than 65 and 65 and older.

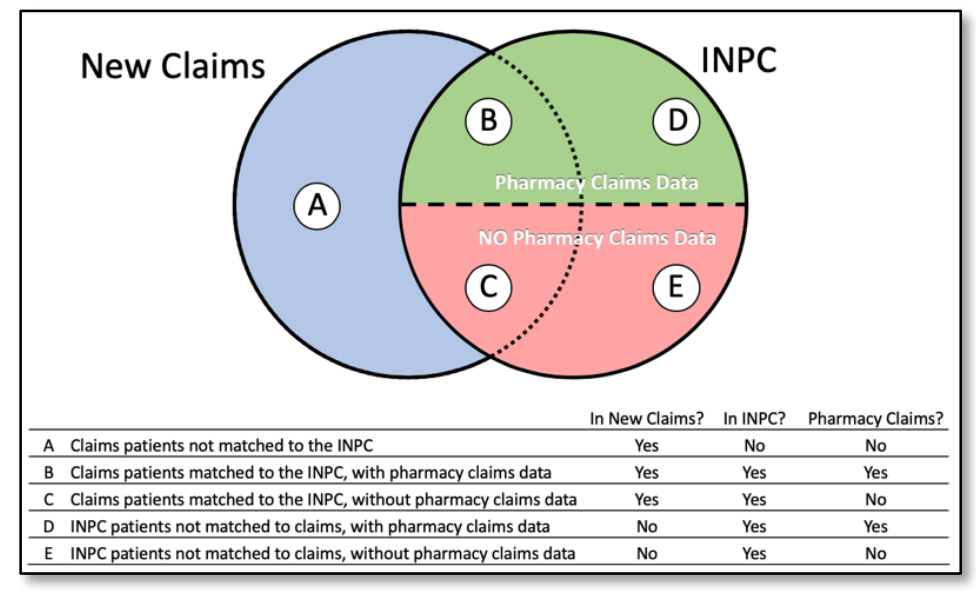

Figure 1: Venn diagram illustrating medication claims data analysis approach. Several subgroups among the INPC will be compared to existing pharmacy claims and new claims data sources.

Phase 2: Investigation of data quality on a clinical use case. These datasets will be used to investigate the current state of the opioid epidemic in Indiana.

Results: With the completion of phase 1, we expect to characterize the current state of claims data across each of the patient groups, and how they influence data quality within the INPC. 
Discussion. The quality and completeness of medication data currently available via the INPC in unclear. Our efforts add clarity to the current status of these datasets, and how they can be augmented for increased research and clinical productivity.

\section{Citations}

[1] Stange, K.C., The problem of fragmentation and the need for integrative solutions. The Annals of Family Medicine. 7(2):100-103, 2009.

[2] McDonald, C. J., Overhage, J. M., Barnes, M., Schadow, G., Blevins, L., Dexter, P. R., ... \& INPC Management Committee. (2005). The Indiana network for patient care: a working local health information infrastructure. Health affairs, 24(5), 1214-1220. 